\title{
ANALYSIS OF ACCURACY OF MODIS BRDF PRODUCT (MCD43 C6) BASED ON MISR LAND SURFACE BRF PRODUCT - A CASE STUDY OF THE CENTRAL PART OF NORTHEAST ASIA
}

\author{
Jian $\mathrm{Li}^{1}$, Shengbo Chen ${ }^{1, *}$, Wenhan Qin ${ }^{1}$, Mike Murefu ${ }^{1}$, Yufei Wang ${ }^{2}$, Yan $\mathrm{Yu}^{1}$, Zhijun Zhen ${ }^{1}$ \\ ${ }^{1}$ College of Geo-Exploration Science and Technology, Jilin University, Changchun, China - OriNatureLi@163.com, \\ chensb@jlu.edu.cn, wenhan.qin@gmail.com, (mikemurefu16, yuyan14, zhenzj16)@ mails.jlu.edu.cn \\ ${ }^{2}$ Centre for Lightning Protection and Disaster Mitigation of Jilin Province, Changchun, China - wangyuf2006@ 126.com
}

\author{
Commission III, ICWG III/IVb
}

KEY WORDS: MODIS BRDF, model accuracy, MISR BRF, vegetation phenology, snow cover

\begin{abstract}
:
EOS/MODIS land surface Bi-directional Reflectance Distribution Function (BRDF) product (MCD43), with the latest version C6, is one of the most important operational BRDF products with global coverage. The core sub-product MCD43A1 stores 3 parameters of the RossThick-LiSparseR semi-empirical kernel-driven BRDF model. It is important for confident use of the product to evaluate the accuracy of bi-directional reflectance factor (BRF) predicted by MCD43A1 BRDF model (mBRF). A typical region in the central part of Northeast Asia is selected as the study area. The performance of MCD43A1 BRDF model is analyzed in various observation geometries and phenological phases, using Multi-angle Imaging SpectroRadiometer (MISR) land-surface reflectance factor product (MILS_BRF) as the reference data. In addition, MODIS products MCD12Q1 and MOD/MYD10A1 are used to evaluate the impacts of land cover types and snow covers on the model accuracy, respectively. The results show an overall excellent performance of MCD43A1 in representing the anisotropic reflectance of land surface, with root mean square error (RMSE) of 0.0262 and correlation coefficient $(R)$ of 0.9537 , for all available comparable samples of MILS_BRF and mBRF pairs. The model accuracy varies in different months, which is related to the phenological phases of the study area. The accuracy for pixels labelled as 'snow' by MCD43 is obviously low, with RMSE/R of $0.0903 / 0.8401$. Ephemeral snowfall events further decrease the accuracy, with RMSE/ $R$ of $0.1001 / 0.7715$. These results provide meaningful information to MCD43 users, especially those, whose study regions are subject to phenological cycles as well as snow cover and change.
\end{abstract}

\section{INTRODUCTION}

The MODIS Bi-directional Reflectance Distribution Function (BRDF) Product (MCD43) is of the characteristics of high temporal resolution, high spatial resolution, full optical spectral coverage as well as global imaging. It is currently one of the most important operational data sources of land surface BRDF. The MCD43 implements the RossThick-LiSparseR semiempirical BRDF (RTLSR) model and the core sub-product MCD43A1 stores 3 parameters of RTLSR model derived from 16-day high-quality land surface reflectance data (Strahler et al., 1999). The latest version of MCD43A1 is Collection 6 (C6), with a nominal spatial resolution of $500 \mathrm{~m}$. MCD43A1 C6 is produced daily and uses the ninth day of the 16 days as the nominal retrieval date of the product.

A large number of validation and accuracy analysis works have been carried out on RTLSR model and MCD43A1 products (Hu et al., 1997; Huang et al., 2013; Jin et al., 2003; Shuai et al., 2008). These works show that the RTLSR model has a wide range of adaptability, MCD43A1 products have good consistency for a long period, and its accuracy can meet the requirements of many applications and researches.

Most of these efforts focus on the growing season of vegetation. In contrast, validation work on the dormant season and snow cover still need more attention. The performance of the MCD43 product was specifically studied during dormant season and snow cover (Wang, 2011; Wang et al., 2014), however, these researches mainly presented the results about albedo, instead of accuracy of BRF on different observation geometries. Independent validation of MCD43A1 C6 is just beginning. The performance of MCD43A1 C6 was evaluated using Landsat data and MODIS surface reflectances from both Terra and Aqua satellite (Che et al., 2017). Though their conclusions are convincing, their results cannot represent the performance of MCD43A1 C6 model in the directions along the sub-satellite track, particularly within principal plane and its vicinity.

The Multi-angle Imaging Spectroradiometer (MISR), also boarded on the Terra satellite, observes the Earth's surface semi-simultaneously from nine discrete view angles along the sub-satellite track. Research demonstrated that MISR agrees to the ground measurements to within $\pm 1 \%$ in all four bands and has also shown long-term temporal stability (Czapla-Myers et al., 2014). Investigation indicates that both MODIS and MISR calibration of the reflective solar bands have been well maintained (Wu et al., 2014). Therefore, MISR land surface bidirectional reflectance factor (BRF) product (MILS_BRF) is also commonly used to test the model accuracy of MCD43 products (Chen et al., 2008; Fang et al., 2004; Lallart et al., 2008; Lucht, 1998; Lucht and Lewis, 2000; Pinty et al., 2011). However, few researches pay attention to this issue from the perspective of different phenological phases of vegetation and snow cover events.

The objective of this study is to evaluate the performance of the MCD43A1 C6 on different observation geometries based on 
MILS_BRF, and to preliminarily analyze phenological phase and snowfall event impacts on the accuracy of the product.

\section{DATA AND METHODS}

\subsection{The Study Area}

The study area, about $6.7 \times 10^{4} \mathrm{~km}^{2}$, is located in the central part of Northeast Asia (Figure 1) with temperate continental monsoon climate. The rainy season is concentrated in the summer from June to August. In winter, accumulated snow cover occurs in most parts of the study area. The vegetation of the study area has notable phenological cycles. The period from April to October is the vegetation-growing season, and from November to March is the vegetation-dormant season. The types of land use and cover of the study area nearly include all major types of the International Geosphere-Biosphere Programme (IGBP) classification system (Figure 1(b)). The north-western part of the study area is of relatively flat terrain and an agricultural area significantly affected by human activities. The central and eastern parts are dominated by forestcovered mountains. Cities, towns and villages of different sizes scatter all over the study area.
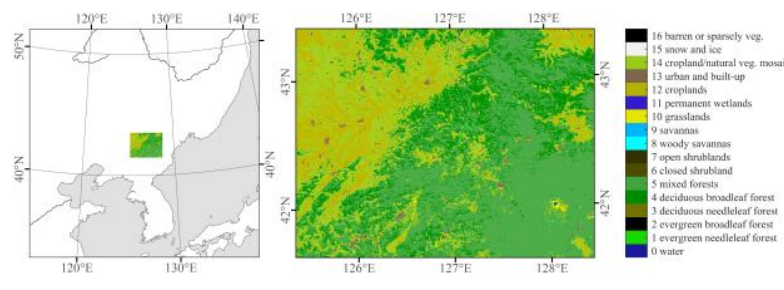

Figure 1 . The study area and its land use and cover types

\subsection{Data}

MODIS products used in this study include BRDF/Albedo product (MCD43) (Schaaf and Wang, 2015), snow cover products (MOD10A1 and MYD10A1) (Hall and Rigss, 2016), and land cover and use product (MCD12Q1) (NASA-LPDAAC, 2017). The spatial resolution of this products is $500 \mathrm{~m}$. The MILS_BRF is used as reference data in this study (MISRScience-Team, 2015). The spatial resolution of this product is $1.1 \mathrm{~km}$. Both MODIS and MISR data cover the period of 20112015 .

\subsection{Methods}

2.3.1 Spatio-temporal co-registration and data selection. The MISR and MODIS data are projected onto customized accurately aligned grids of $1 \mathrm{~km} \times 1 \mathrm{~km}$ and $500 \mathrm{~m} \times 500 \mathrm{~m}$, respectively, both in Albers equal-area conic projection, to achieve spatial matching of 1 MISR pixel with $2 \times 2$ MODIS pixels.

There are a large number of data gaps in each orbit of MISR data. In order to suppress the influence of random noise, the area proportion of the effective BRF data of each orbit of MISR product within the study area is calculated. Dates with an effective data area proportion less than $10 \%$ do not participate in the following analysis. A total of 110 days of MISR data were selected from the period of 2011-2015. The MILS_BRF data with good quality $(\mathrm{QA}=0)$ participate in the comparison.
The MCD43A1 model parameters are linearly aggregated from $2 \times 2$ pixels into 1 pixel. Only the aggregated pixel with all $2 \times 2$ original pixels with best retrieval quality $(\mathrm{QA}=0)$ participates the following analysis.

The MOD10A1/MYD10A1 data are optimally combined at their original spatial resolution of $500 \mathrm{~m}$, i.e., the data with higher quality of the two observations per day are selected. Then the same method as MCD43A1 is used to perform data aggregation and only data with the best quality $(\mathrm{QA}=0)$ participate in the following analysis. The merged result is named as MCD10A1, hereinafter.

2.3.2 Accuracy analysis of MCD43A1 model. For MILS_BRF, $r_{\text {MISR }}\left(\Phi_{i}, \mathrm{P}_{\mathrm{j}}, T_{k}\right)$ is regarded as 'true' BRF value of land-surface pixel $\mathrm{P}_{i}$ in nominal observation date $T_{k}$ and observation geometry $\Phi_{i}$, where $\Phi_{i} \equiv\left(\theta_{s i}, \theta_{v i}, \varphi_{i}\right)$ is the triple composed of solar zenith angle (SZA), view zenith angle (VZA), and view-sun relative azimuth angle (RAA). Correspondingly, $r_{M O D}\left(\Phi_{i}, \mathrm{P}_{\mathrm{j}}, T_{k}\right)$ is defined as the BRF value predicted by MCD43A1 model:

$$
\begin{aligned}
& r_{M O D}\left(\Phi_{i}, \mathrm{P}_{\mathrm{j}}, T_{k}\right)= \\
& f_{\text {iso }}\left(\mathrm{P}_{\mathrm{j}}, T_{k}\right)+f_{v o l}\left(\mathrm{P}_{\mathrm{j}}, T_{k}\right) k_{v o l}\left(\Phi_{i}\right)+f_{g e o}\left(\mathrm{P}_{\mathrm{j}}, T_{k}\right) k_{g e o}\left(\Phi_{i}\right)
\end{aligned}
$$

where $f_{\text {iso }}, f_{\text {vol }}$, and $f_{\text {geo }}$ are the coefficients of isotropic scattering kernel, volumetric scattering kernel $k_{v o l}$, and geometric-optical surface scattering kernel $k_{\text {geo }}$, respectively. Root mean square error $e_{r m s}$, correlation coefficient $R$ and bias $b$ are used to evaluate the performance of MCD43A1 model for BRF prediction:

$$
\begin{aligned}
& e_{r m s}=\sqrt{\frac{1}{N} \sum_{n=1}^{N}\left(\left(r_{M O D}(n)-r_{M I S R}(n)\right)^{2}\right.} \\
& R=\frac{\sum_{n=1}^{N}\left(r_{M O D}(n)-\overline{r_{M O D}}\right)\left(r_{M I S R}(n)-\overline{r_{M I S R}}\right)}{\sqrt{\sum_{n=1}^{N}\left(r_{M O D}(n)-\overline{r_{M O D}}\right)^{2} \sum_{i=1}^{N}\left(r_{M I S R}(n)-\overline{r_{M I S R}}\right)^{2}}} \\
& b=\frac{1}{N} \sum_{n=1}^{N}\left(\left(r_{M O D}(n)-r_{M I S R}(n)\right)\right.
\end{aligned}
$$

where $n \equiv\left(\Phi_{i}, \mathrm{P}_{\mathrm{j}}, T_{k}\right)$ and $\bar{r}=\frac{1}{N} \sum_{n=1}^{N} r(n)$.

2.3.3 Groups of observation geometry for analysis of MCD43A1 model performance. For all reference data, i.e. MILS_BRF data, their view zenith angles and view-sun relative azimuth angles are divided into 9 and 3 groups, respectively.

View zenith angles are grouped by 9 MISR cameras, Df, Cf, Bf, Af, An, Aa, Ba, Ca and Da, representing nominal VZAs of $70.5^{\circ},-60.0^{\circ},-45.6^{\circ},-26.1^{\circ}, 0^{\circ}, 26.1^{\circ}, 45.6^{\circ}, 60.0^{\circ}, 70.5^{\circ}$. The view-sun relative azimuths are divided into three groups: principal plane $(\mathrm{PP}) \pm 30^{\circ}$ (near PP directions, nPP), cross principal plane $(\mathrm{CP}) \pm 30^{\circ}$ (near $\mathrm{CP}$ directions, $\mathrm{nCP}$ ) and other (XP) azimuths. Their specific angular ranges are $\left(0^{\circ}, 30^{\circ}\right) \cup$ 
$\left(150^{\circ}, 180^{\circ}\right),\left(60^{\circ}, 120^{\circ}\right)$, and $\left[30^{\circ}, 60^{\circ}\right] \cup\left[120^{\circ}, 150^{\circ}\right]$, respectively.

\section{RESULT ANALYSIS}

\subsection{Overall Accuracy of MCD43A1 model}

According to the data selection method of this paper, 878,337 groups of MCD43A1 RTLSR model parameters and corresponding 7,905,033 MILS_BRF data were found in the study area and the period of 2011-2015. These data cover forests, grass, crop, urban pixels and their annual phenological cycles. For all the available comparable data, the MCD43A1 product shows overall excellent performance, with $e_{r m s} / R=$ $0.0262 / 0.9537$, in representing the anisotropic reflectance of land surface (Figure 2(d)).

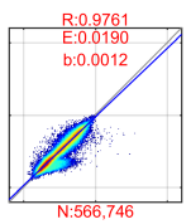

(a)

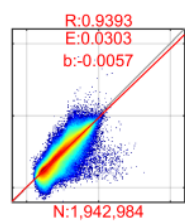

(b)

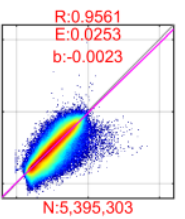

(c)

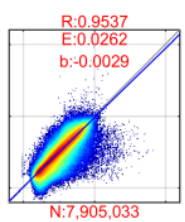

(d)
Figure 2. Overall accuracy of MCD43A1 model.

The 4 sub-figures of Figure 2 represent model accuracy within directions of $\mathrm{nCP}, \mathrm{nPP}, \mathrm{XP}$, and all RAAs, respectively. The horizontal axis of each sub-figure represents the value of MILS_BRF, and the vertical axis represents the value of BRF predicted by MCD43A1. The inner (outer) frame lines represent the range of values $[0,1]([-0.1,1.1])$. The gray slash is $1: 1$ line, and the color line is the regression line. $\mathrm{R}, \mathrm{E}$, and $\mathrm{b}$ are the correlation coefficient, RMSE, and bias of model respectively, and $\mathrm{n}$ is the number of samples.

Moreover, the MCD43A1 model shows high accuracies for all the 4 major land cover types of the study area, i.e. forest, grass/crop land, urban, and the mixed, with $e_{r m s}<0.0265$ (the mixed land cover type with the largest error), $R>0.9340$ (urban with the lowest correlation coefficient).

\subsection{The Impact of Observation Geometry on the Accuracy of MCD43A1 Model}

At different RAAs, the MCD43A1 model shows highest accuracy in nCP directions with $e_{r m s} / R=0.0190 / 0.9761$ (Figure 2(a)) and lowest accuracy in nPP directions with $e_{r m s} / R=0.0303 / 0.9393$ (Figure 2(b)). The accuracy in XP directions is between that of $\mathrm{nCP}$ and $\mathrm{nPP}$ (Figure 2(c)).

For all the available comparable samples, the accuracy of backward scattering (RAZ > $90^{\circ}$, using the RAZ definition of MILS_BRF, hereinafter) is better than that of forward scattering $\left(\mathrm{RAZ}<90^{\circ}\right)$, and the same is true in the $\mathrm{nPP}$ and $\mathrm{XP}$ direction subsets. In contrast, the forward/backward scattering accuracies of the nCP direction subset are similar, with $e_{r m s} / R=$ $0.0154 / 0.9689$ and 0.0233/0.9719, respectively. (Figure 3(a)).

At different VZAs, the large model errors lie in the direction of the large VZAs $\left( \pm 70.5^{\circ}\right)$, and the model errors for forward scattering are mainly concentrated on the two VZAs of $-70.5^{\circ}$ and $-60^{\circ}$ (camera Df and Cf). For VZAs of $45.6^{\circ}, 26.1^{\circ}$, and $0^{\circ}$, the errors of model of forward scattering (camera Bf, Af, An-) are smaller than those of the corresponding backward scattering (camera Ba, Aa, An+). The bias of the model is -0.0237 for the VZA of $-70.5^{\circ}$ (camera Df), which greatly exceeds the bias range of other 9 VZAs $( \pm 0.008)$.

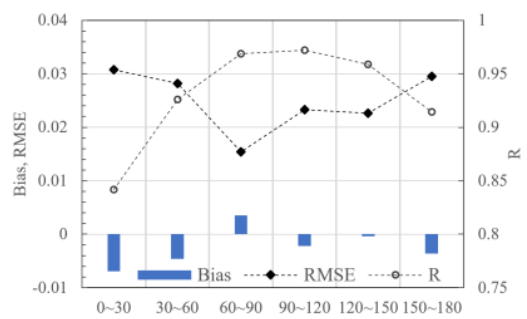

(a)

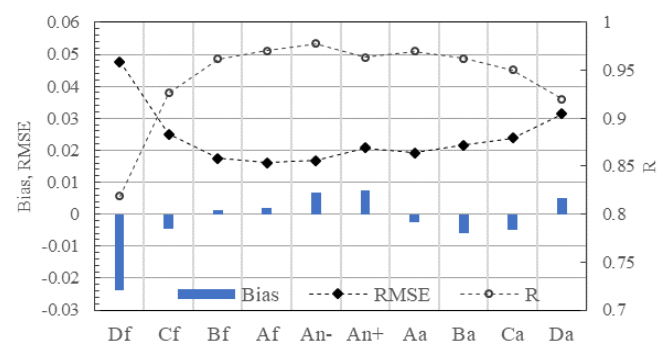

(b)

Figure 3. The accuracy of MCD43A1 model on different (a) RAAs and (b) VZAs.

\subsection{The Impact of Phenological Phases on the Accuracy of MCD43A1 Model}
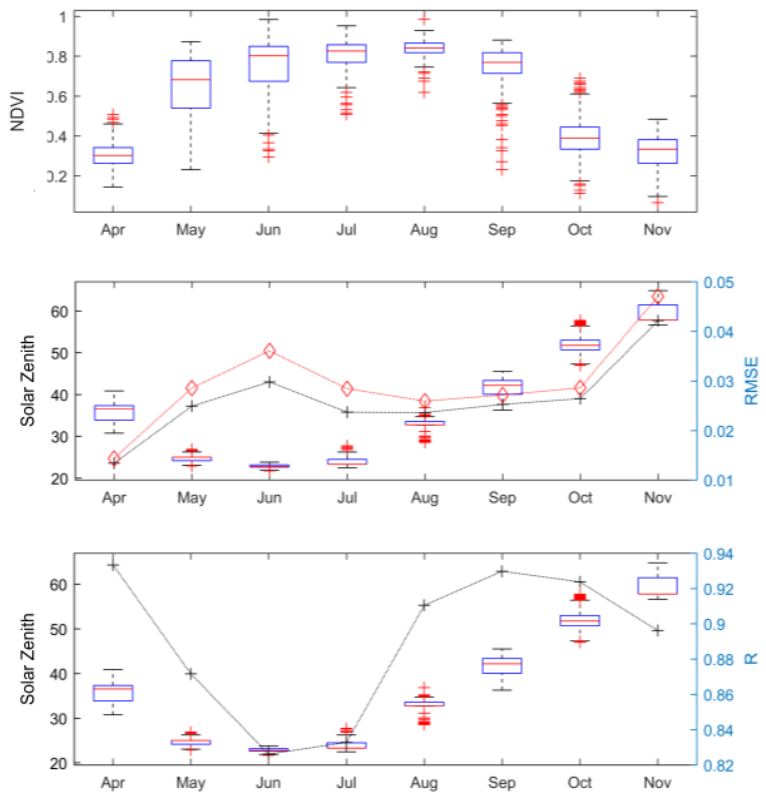

Figure 4. The impact of phenological phases on the accuracy of MCD43A1 model.

In row 2 of Figure 4, lines with + and $\diamond$ represent $e_{r m s}$ and $e_{r m s} / R$, respectively. In row 3 of Figure 4 , the line with + represents $R$. Data sources of box plots are labelled as the name of vertical axis.

The accuracy of the model varies significantly in different months. For all the available comparable pixels of MCD43A1 
and MILS_BRF, April has the highest accuracy $\left(e_{r m s} / R=\right.$ $0.0144 / 0.9227)$ and November has the maximum $e_{r m s}(0.0358)$. For common pixel of each month of the growing season (from April to October), the accuracy of April is still the highest ( $e_{r m s} / R=0.0134 / 0.9333$ ) and June has the lowest accuracy $\left(e_{r m s} / R=0.0298 / 0.8261\right)$.

The variation of accuracy of the model is related to the phenological phase of the study area. In general, the error of MCD43A1 model, accompanying phenological phase change of the study area, experiences a process of "continuous rise, decline, stability, and rapid rise". (Figure 4).

\subsection{The Impact of Snow Cover on the Accuracy of MCD43A1 Model}

3.4.1 The accuracy of MCD43A1 model for snow pixel. During the 16-day window for the inversion of MCD43A1 BRDF model parameters, if on the nominal production date (day 9) the MCD10A1 pixel are covered by snow, other days with snow covered pixels will be selected together for retrieval of model parameters, and vice versa (Wang et al., 2013). There are only 64 groups samples with MCD43A1 parameters retrieved as high quality snow pixel. The accuracy of MCD43A1 of these samples, with $e_{r m s} / R=0.0903 / 0.8401$, is obviously lower than that of the same pixels when free of snow cover, with $e_{r m s} / R=0.0242 / 0.9505$. The BRF prediction error of MCD43A1 model with parameters retrieved as snow pixel are mainly concentrated in $+60.0^{\circ}$ and $+70.50^{\circ}$ (MISR camera $\mathrm{Ca}$ and Da). Figure 5 shows the model predicting BRF (magenta) and MILS_BRF (cyan) at each observation zenith angle, where the bold lines represent the average value, and left panel for snow retrieval and right panel for snow-free retrieval.
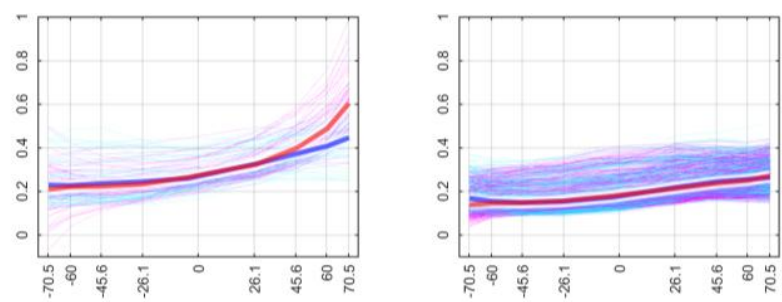

Figure 5. Comparison of accuracy of MCD43A1 model for snow pixels with that for the same pixels when free of snow

3.4.2 The impact of ephemeral snow cover on accuracy of MCD43A1 model. The ephemeral snow cover is defined here as the situation that on a nominal comparison date, the MCD10A1 snow cover proportion of a pixel is not less than $30 \%$, but the MCD43A1 model parameters are not retrieved as snow pixels.

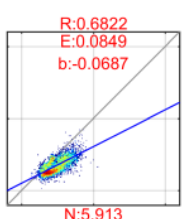

(a)

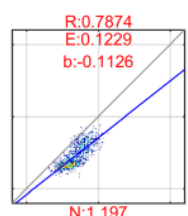

(b)

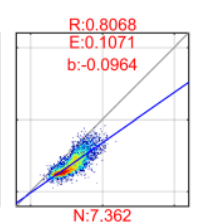

(c)

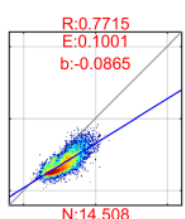

(d)
Figure 6. The impact of ephemeral snow cover event on accuracy of MCD43A1 model
Ephemeral snow cover event decrease the model accuracy significantly $\left(e_{r m s} / R=0.1001 / 0.7715\right)$. Under ephemeral snow cover event, the model accuracy for all 4 major land cover types of the study area reduces obviously. Figure 6 shows the details for (a) forest, (b) grass/cropland, (c) mixed, and (d) all land cover types.

\section{DISCUSSION}

MILS_BRF is of significant importance in testing the performance of MCD43A1 BRDF model. MILS_BRF and MCD43A1 products are synchronous, have comparable spatial resolution, and possess 4 similar spectral channels, whilst on the other hand, the MILS_BRF has wider range of sun-view relative azimuth angle and view zenith angle, and, particularly, the observation capability in the directions along orbit and near principal plane. This provides the possibility of testing extrapolating accuracy of MCD43A1 BRDF model in these crucial observation geometries. In contrast, results of analysis based on Aqua/MODIS and Landsat data (Che et al., 2017) can only represent the performance of MCD43A1 C6 BRDF model in the directions of cross orbit or cross principal plane. From this perspective, results of this paper complement a larger part of the whole picture of testing MCD43A1 C6 BRDF model in hemisphere space of observation.

Admittedly, the MILS_BRF itself is a satellite retrieval product and there exists uncertainty compared to ground-based BRF observations. However, research (Czapla-Myers et al., 2014) demonstrated that MISR agrees to the ground measurements to within $\pm 1 \%$ in all four bands and has also shown long-term temporal stability. Also, investigation (Wu et al., 2014) indicates that both MODIS and MISR calibration of the reflective solar bands have been well maintained. These results provide confidence for MILS_BRF to be used as comparison reference.

MISR is not uniform in the sampling of the whole hemisphere, and there exists significant spatial and temporal variation of MILS_BRF of different observational geometry. Therefore, complete evaluation of MCD43A1 in hemisphere space of observation requires data from more view angles.

\section{CONCLUSIONS}

In this study, a typical region in the central part of Northeast Asia is selected as the study area and the performance of MCD43A1 C6 BRDF model is analysed in various observation geometries and phenological phases, using the Multi-angle Imaging SpectroRadiometer (MISR) land-surface reflectance factor (BRF) product (MILS_BRF) as the reference data. In addition, the impacts of land cover types and snow covers on the model accuracy are evaluated using MODIS land cover type product and snow cover products.

The results show the overall excellent performance of MCD43A1 C6 product to represent the anisotropic reflectance of land surface with root mean square error (RMSE) of 0.0262 and correlation coefficient (R) of 0.9537 for all available comparable samples of MILS_BRF and BRF predicted by MCD43A1 model. The model accuracy varies in different months, which is related to phenological phases of the study area. The accuracy of MCD43A1 model of pixels labelled as 'snow' by MCD43 is obviously low with RMSE/R of 
0.0903/0.8401. Ephemeral snow fall events further decrease the accuracy of MCD43A1 model with RMSE/R of $0.1001 / 0.7715$, though the MCD43A1 model parameters are labelled as 'best quality, full inversion' and 'snow free'. These results provide meaningful information to MCD43 users, especially those, whose study regions are subject to phenological cycles as well as snow cover and change.

\section{ACKNOWLEDGEMENTS}

This work is supported by (1) the program for JLU Science and Technology Innovative Research Team (No. 2017TD-26) which is funded by the Fundamental Research Funds for the Central Universities, China and (2) the Plan for Changbai Mountain Scholars of Jilin Province, China (JJLZ[2015]54).

\section{REFERENCES}

Che, X., Feng, M., Sexton, J., Channan, S., Yang, Y., Sun, Q., 2017. Assessment of MODIS BRDF/Albedo Model Parameters (MCD43A1 Collection 6) for Directional Reflectance Retrieval. Remote Sens. 9(11), pp. 1122-1137.

https://doi.org/10.3390/rs9111123.

Chen, Y., Wang, J., Liang, S., Wang, D., Ma, B., Bo, Y., 2008. The Bidirectional Reflectance Signature of Typical Land Surfaces and Comparison of MISR and MODIS BRDF Products. In IEEE IGARSS 2008, Vol. 3, pp. III1099-1102. https://doi.org/10.1109/IGARSS.2008.4779546.

Czapla-Myers, J., Thome, K., Anderson, N., Biggar, S., 2014. The absolute radiometric calibration of Terra imaging sensors: MODIS, MISR, and ASTER. In Earth Observing Systems XIX, Vol. 9218, pp. Y1-10. https://doi.org/10.1117/12.2062529.

Fang, H., Liang, S., Chen, M., Walthall, C., Daughtry, C., 2004. Statistical comparison of MISR, ETM plus and MODIS land surface reflectance and albedo products of the BARC land validation core site, USA. Int. J. Remote Sens. 25(2), pp. 409422. https://doi.org/10.1080/0143116031000101666.

Hall, D.K., Rigss, G.A., 2016. MODIS/Terra Snow Cover Daily L3 Global 500m Grid, Version 6. NASA National Snow and Ice Data Center DAAC, Boulder, Colorado USA.

https://doi.org/10.5067/MODIS/MOD10A1.006.

Hu, B., Lucht, W., Li, X., Strahler, A.H., 1997. Validation of kernel-driven semiempirical models for the surface bidirectional reflectance distribution function of land surfaces. Remote Sens. Environ. 62(3), pp. 201-214. https://doi.org/10.1016/S00344257(97)00082-5.

Huang, X., Jiao, Z., Dong, Y., Zhang, H., Li, X., 2013. Analysis of BRDF and Albedo Retrieved by Kernel-Driven Models Using Field Measurements. IEEE J. Sel. Topics Appl. Earth Observ. Remote Sens. 6(1), pp. 149-161.

https://doi.org/10.1109/JSTARS.2012.2208264.

Jin, Y., Schaaf, C.B., Woodcock, C.E., Gao, F., Li, X., Strahler, A.H., Lucht, W., Liang, S., 2003. Consistency of MODIS surface bidirectional reflectance distribution function and albedo retrievals: 2. Validation. J. Geophys. Res.: Atmos. 108(D5), pp. 1-15. https://doi.org/10.1029/2002JD002804.
Lallart, P., Kahn, R., Tanre, D., 2008. POLDER2/ADEOSII, MISR, and MODIS/Terra reflectance comparisons. J. Geophys. Res.: Atmos. 113(D14), pp. S02(01-08). https://doi.org/10.1029/2007JD009656.

Lucht, W., 1998. Expected retrieval accuracies of bidirectional reflectance and albedo from EOS-MODIS and MISR angular sampling. J. Geophys. Res.: Atmos. 103(D8), pp. 8763-8778. https://doi.org/10.1029/98JD00089.

Lucht, W., Lewis, P., 2000. Theoretical noise sensitivity of BRDF and albedo retrieval from the EOS-MODIS and MISR sensors with respect to angular sampling. Int. J. Remote Sens. 21(1), pp. 81-98. https://doi.org/10.1080/014311600211000.

MISR-Science-Team, 2015. Terra/MISR Level 2 Land Surface Data, version 2. NASA ASDC, Hampton, VA, USA. https://doi.org/10.5067/Terra/MISR/MIL2ASLS_L2.002.

NASA-LP-DAAC, 2017. MODIS Land Cover Type Yearly L3 Global 500 m SIN Grid (MCD12Q1). NASA EOSDIS LPDAAC, USGS EROS Center, Sioux Falls, South Dakota https://lpdaac.usgs.gov/dataset_discovery/modis/modis_product s_table/mcd $12 \mathrm{q} 1$.

Pinty, B., Taberner, M., Haemmerle, V.R., Paradise, S.R., Vermote, E., Verstraete, M.M., Gobron, N., Widlowski, J.L., 2011. Global-Scale Comparison of MISR and MODIS Land Surface Albedos. J. Climate 24(3), pp. 732-749. https://doi.org/10.1175/2010JCLI3709.1.

Schaaf, C., Wang, Z., 2015. MCD43A1 MODIS/Terra+Aqua BRDF/Albedo Model Parameters Daily L3 Global - 500m V006. NASA EOSDIS LPDAAC. https://doi.org/10.5067/MODIS/MCD43A1.006.

Shuai, Y., Schaaf, C.B., Strahler, A.H., Liu, J., Jiao, Z., 2008. Quality assessment of BRDF/albedo retrievals in MODIS operational system. Geophys. Res. Lett. 35(5), pp. 1-5. https://doi.org/10.1029/2007gl032568.

Strahler, A.H., Muller, J.-P., Lucht, W., Schaaf, C.B., Tsang, T., Gao, F., Li, X., Lewis, P., Barnsley, M.J., 1999. MODIS BRDF/Albedo Product: Algorithm Theoretical Basis Document Version 5.0.

Wang, Z., 2011. The MODIS reflectance anisotropy and albedo of dormant and snow-covered canopies., Boston University. ProQuest Dissertations Publishing.

Wang, Z., Schaaf, C., Román, M., 2013. MODIS Collection 6 BRDF/Albedo: Status and Updates.

https://modis.gsfc.nasa.gov/sci_team/meetings/201304/presentat ions/plenary/wang.pdf. (Nov 11, 2017).

Wang, Z., Schaaf, C.B., Strahler, A.H., Chopping, M.J., Román, M.O., Shuai, Y., Woodcock, C.E., Hollinger, D.Y., Fitzjarrald, D.R., 2014. Evaluation of MODIS albedo product (MCD43A) over grassland, agriculture and forest surface types during dormant and snow-covered periods. Remote Sens. Environ. 140, pp. 60-77. https://doi.org/10.1016/j.rse.2013.08.025.

Wu, A.S., Angal, A., Xiong, X.X., 2014. Comparison of coincident MODIS and MISR reflectances over the 15-year period of EOS Terra. In Earth Observing Systems XIX, Vol. 9218, pp. W1-13. https://doi.org/10.1117/12.2061117. 